\title{
Jarlskog Invariant of the Neutrino Mapping Matrix
}

\author{
R. Friedberg ${ }^{1}$ and T. D. Lee Le $^{1,2}$ \\ 1. Physics Department, Columbia University \\ New York, NY 10027, U.S.A. \\ 2. China Center of Advanced Science and Technology (CCAST/World Lab.) \\ P.O. Box 8730, Beijing 100080, China
}

\begin{abstract}
The Jarlskog Invariant $J_{\nu-\text { map }}$ of the neutrino mapping matrix is calculated based on a phenomenological model which relates the smallness of light lepton masses $m_{e}$ and $m_{1}$ (of $\nu_{1}$ ) with the smallness of $T$ violation. For small $T$ violating phase $\chi_{l}$ in the lepton sector, $J_{\nu-m a p}$ is proportional to $\chi_{l}$, but $m_{e}$ and $m_{1}$ are proportional to $\chi_{l}^{2}$. This leads to $J_{\nu-m a p} \cong \frac{1}{6} \sqrt{\frac{m_{e}}{m_{\mu}}}+O\left(\sqrt{\frac{m_{e} m_{\mu}}{m_{\tau}^{2}}}\right)+O\left(\sqrt{\frac{m_{1} m_{2}}{m_{3}^{2}}}\right)$. Assuming $\sqrt{\frac{m_{1} m_{2}}{m_{3}^{2}}}<<\sqrt{\frac{m_{e}}{m_{\mu}}}$, we find $J_{\nu-m a p} \cong 1.16 \times 10^{-2}$, consistent with the present experimental data.
\end{abstract}

PACS: $14.60 . \mathrm{Pq}, \quad 11.30 . \mathrm{Er}$

Key words: Jarlskog invariant, hidden symmetry, neutrino mapping matrix 


\section{Introduction}

In a recent paper[1], we proposed a phenomenological model in which the smallness of light quark masses $m_{d}$ and $m_{u}$ are related to the smallness of $T$ violation. Thus, when the relevant $T$ violating phase $\chi_{q}$ in the quark sector is nonzero but small, $m_{d}$ and $m_{u}$ are proportional to $\chi_{q}^{2}$. On the other hand, the Jarlskog invariant $J_{C K M}$ of the CKM matrix depends linearly on $\chi_{q}$, which leads to a relation

$$
\begin{aligned}
J_{C K M} \cong A \lambda^{3}[ & \left.\sqrt{\frac{m_{d} m_{s}}{m_{b}^{2}}}+O\left(\sqrt{\frac{m_{u} m_{c}}{m_{t}^{2}}}\right)\right] \\
& \cong 3 \times 10^{-5}
\end{aligned}
$$

where $A \cong 0.818$ and $\lambda \cong 0.227$ are the Wolfenstein parameters. In this paper, we continue the model analysis for the lepton sector. As we shall see, in the small $T$ violation limit the corresponding Jarlskog invariant $J_{\nu-m a p}$ of the neutrino mapping matrix is related to the lepton masses by

$$
J_{\nu-m a p} \cong \frac{1}{6} \sqrt{\frac{m_{e}}{m_{\mu}}}+O\left(\sqrt{\frac{m_{e} m_{\mu}}{m_{\tau}^{2}}}\right)+O\left(\sqrt{\frac{m_{1} m_{2}}{m_{3}^{2}}}\right)
$$

where $m_{1}, m_{2}$ and $m_{3}$ are masses of $\nu_{1}, \nu_{2}$ and $\nu_{3}$. Assuming

$$
\frac{m_{1} m_{2}}{m_{3}^{2}}<<\frac{m_{e}}{m_{\mu}}
$$

we find

$$
J_{\nu \text { map }} \cong \frac{1}{6} \sqrt{\frac{m_{e}}{m_{\mu}}} \cong 1.16 \times 10^{-2}
$$

consistent with the present ( $1 \sigma$-deviation) experimental limit[2]

$$
J_{\nu-\text { map }} \leq 4.2 \times 10^{-2} .
$$

Thus, an improvement of a factor 4 of the experimental accuracy could yield a meaningful test of the model.

The underlying reasoning of our analysis is a spontaneous $T$ violation field theory model $[3,4]$, in which the Higgs field responsible for $T$ violation belongs to the same family of scalar fields that generates masses for light quarks and 
light leptons. However, in this paper and Ref. 1, we restrict our discussions only to a phenomenological analysis of the relevant mass matrices. As in Ref. 1, let $l_{i}(\downarrow)$ and $l_{i}(\uparrow)$ be the hypothetical lepton states "diagonal" in $W^{ \pm}$transitions:

$$
l_{i}(\downarrow) \rightleftharpoons l_{i}(\uparrow)+W^{-}
$$

and

$$
l_{i}(\uparrow) \rightleftharpoons l_{i}(\downarrow)+W^{+}
$$

with $i=1,2,3$. Their electric charges in units of $e$ are -1 for $l_{i}(\downarrow)$, and 0 for $l_{i}(\uparrow)$. For each of these triplets, there exists a $3 \times 3$ mass matrix $M(l)$ with the corresponding mass operator $\mathcal{M}(l)$ given by

$$
\mathcal{M}\left(l_{\downarrow}\right)=\left(\bar{l}_{1}(\downarrow), \bar{l}_{2}(\downarrow), \bar{l}_{3}(\downarrow)\right) M\left(l_{\downarrow}\right)\left(\begin{array}{c}
l_{1}(\downarrow) \\
l_{2}(\downarrow) \\
l_{3}(\downarrow)
\end{array}\right),
$$

and

$$
\mathcal{M}\left(l_{\uparrow}\right)=\left(\bar{l}_{1}(\uparrow), \bar{l}_{2}(\uparrow), \bar{l}_{3}(\uparrow)\right) M\left(l_{\uparrow}\right)\left(\begin{array}{c}
l_{1}(\uparrow) \\
l_{2}(\uparrow) \\
l_{3}(\uparrow)
\end{array}\right)
$$

in which the related Dirac field operator $\psi$ and its Hermitian conjugate $\psi^{\dagger}$ are given by

$$
\psi_{i \downarrow}=l_{i}(\downarrow), \quad \psi_{i \downarrow}^{\dagger} \gamma_{4}=\bar{l}_{i}(\downarrow)
$$

and likewise for the $\uparrow$ sector.

In the zeroth approximation of $T$ invariance, $M\left(l_{\downarrow}\right)$ and $M\left(l_{\uparrow}\right)$ are both real. At the same time, we assume that the mass operator $\mathcal{M}\left(l_{\downarrow}\right)$ satisfies a hidden symmetry

$$
l_{1}(\downarrow) \rightarrow l_{1}(\downarrow)+z, \quad l_{2}(\downarrow) \rightarrow l_{2}(\downarrow)+\eta_{\downarrow} z \quad \text { and } \quad l_{3}(\downarrow) \rightarrow l_{3}(\downarrow)+\xi_{\downarrow} \eta_{\downarrow} z
$$

where $\xi_{\downarrow}, \quad \eta_{\downarrow}$ are $c$-numbers and $z$ is a space-time independent constant element, anticommuting with the Dirac field operators. A similar symmetry is assumed for the $\uparrow$ sector. As shown in Ref. 1, in each sector $\downarrow$ and $\uparrow$, this symmetry yields a zero mass particle state (i.e., $e$ and $\nu_{1}$ ). Thus, we may write the zeroth approximation of $\mathcal{M}\left(l_{\downarrow}\right)$ and $\mathcal{M}\left(l_{\uparrow}\right)$ as

$$
\mathcal{M}_{0}\left(l_{\downarrow}\right)=\alpha_{\downarrow}\left|l_{3}(\downarrow)-\xi_{\downarrow} l_{2}(\downarrow)\right|^{2}+\beta_{\downarrow}\left|l_{2}(\downarrow)-\eta_{\downarrow} l_{1}(\downarrow)\right|^{2}+\gamma_{\downarrow}\left|l_{1}(\downarrow)-\zeta_{\downarrow} l_{3}(\downarrow)\right|^{2}
$$


and

$$
\mathcal{M}_{0}\left(l_{\uparrow}\right)=\alpha_{\uparrow}\left|l_{3}(\uparrow)-\xi_{\uparrow} l_{2}(\uparrow)\right|^{2}+\beta_{\uparrow}\left|l_{2}(\uparrow)-\eta_{\uparrow} l_{1}(\uparrow)\right|^{2}+\gamma_{\uparrow}\left|l_{1}(\uparrow)-\zeta_{\uparrow} l_{3}(\uparrow)\right|^{2}
$$

with the 12 parameters $\alpha_{\downarrow}, \xi_{\downarrow}, \cdots$ all real. The symmetry (1.10) for the $\downarrow$ and $\uparrow$ sectors requires

$$
\xi_{\downarrow} \eta_{\downarrow} \zeta_{\downarrow}=1
$$

and

$$
\xi_{\uparrow} \eta_{\uparrow} \zeta_{\uparrow}=1 .
$$

Consequently, in the approximation of $T$ invariance the model contains

$$
2 \times(6-1)=10
$$

real parameters. On the other hand, in the same approximation, there are 4 nonzero masses of $\mu, \tau, \nu_{2}$ and $\nu_{3}$, In addition the $\nu$-mapping matrix is real, specified by 3 parameters. Thus, the total number of observables are

$$
4+3=7
$$

The difference

$$
10-7=3
$$

gives the three "gauge" degrees of freedom that are needed to specify the orientation of the 3-dimensional hidden frame $\Sigma_{l}$ characterized by its orthonormal axes $\hat{l}_{1}, \hat{l}_{2}$ and $\hat{l}_{3}$, with $\hat{l}_{i}=\hat{l}_{i}(\downarrow)=\hat{l}_{i}(\uparrow)$ and $i=1,2,3$.

In Ref. 1, two of these "gauge" degrees of freedom are eliminated by requiring

$$
\beta_{\downarrow}=\gamma_{\downarrow} \zeta_{\downarrow}^{2} \text { and } \beta_{\uparrow}=\gamma_{\uparrow} \zeta_{\uparrow}^{2}
$$

As we shall see, these two conditions have a simple geometrical interpretation. Let $\Sigma_{\nu}$ be the reference frame with its base vectors defined by the physical state-vectors $\hat{\nu}_{1}, \hat{\nu}_{2}$ and $\hat{\nu}_{3}$. Likewise, let $\Sigma_{e}$ be the corresponding reference frame whose base vectors are the physical state-vectors $\hat{e}, \hat{\mu}$ and $\hat{\tau}$. As we shall see, the two conditions in (1.18) are equivalent to choosing the orientation of the $\hat{l}_{1}$ axis in the hidden frame $\Sigma_{l}$ to be perpendicular to $\hat{\nu}_{3}$ and $\hat{\tau}$; i.e.,

$$
\hat{l}_{1} \| \hat{\nu}_{3} \times \hat{\tau} \text {. }
$$


To establish this relation, we follow the same notations and steps used in Ref. 1 for the quark sector. Define four angular variables $\theta_{\downarrow}, \phi_{\downarrow}$ and $\theta_{\uparrow}, \phi_{\uparrow}$ by

$$
\begin{gathered}
\xi_{\downarrow}=\tan \phi_{\downarrow}, \quad \xi_{\uparrow}=\tan \phi_{\uparrow} \\
\eta_{\downarrow}=\tan \theta_{\downarrow} \cos \phi_{\downarrow} \text { and } \eta_{\uparrow}=\tan \theta_{\uparrow} \cos \phi_{\uparrow} .
\end{gathered}
$$

It is useful to introduce a subscript $s$, with

$$
s=\downarrow \text { or } \uparrow .
$$

The eigenstates of the zeroth order mass operator $M_{0}\left(l_{s}\right)$ are

$$
\begin{aligned}
\epsilon_{s} & =\left(\begin{array}{l}
\cos \theta_{s} \\
\sin \theta_{s} \cos \phi_{s} \\
\sin \theta_{s} \sin \phi_{s}
\end{array}\right), \\
p_{s} & =\left(\begin{array}{l}
-\sin \theta_{s} \\
\cos \theta_{s} \cos \phi_{s} \\
\cos \theta_{s} \sin \phi_{s}
\end{array}\right)
\end{aligned}
$$

and

$$
P_{s}=\left(\begin{array}{l}
0 \\
-\sin \phi_{s} \\
\cos \phi_{s}
\end{array}\right)
$$

with their corresponding eigenvalues given by

$$
\begin{gathered}
\lambda\left(\epsilon_{s}\right)=0 \\
\lambda\left(p_{s}\right)=\beta_{s}\left[1+\eta_{s}^{2}\left(1+\xi_{s}^{2}\right)\right]
\end{gathered}
$$

and

$$
\lambda\left(P_{s}\right)=\alpha_{s}\left(1+\xi_{s}^{2}\right)+\beta_{s} .
$$

Hence, these state-vectors are the zeroth order physical lepton states with

$$
\begin{gathered}
\epsilon_{\downarrow}=e, \quad p_{\downarrow}=\mu, \quad P_{\downarrow}=\tau, \\
\epsilon_{\uparrow}=\nu_{1}, \quad p_{\uparrow}=\nu_{2}, \quad P_{\uparrow}=\nu_{3} .
\end{gathered}
$$


From (1.23), one sees that the first elements of $P_{\downarrow}$ and $P_{\uparrow}$ are both zero. (The motivation for imposing (1.18) is essentially to achieve this fact which simplifies calculations.) Hence, (1.19) follows.

Let $\left(V_{s}\right)_{0}$ be the $3 \times 3$ unitary matrix that diagonalizes the mass matrix $M_{0}\left(l_{s}\right)$ of (1.11)-(1.12). We have, by using (1.22)-(1.24),

$$
\left(V_{s}\right)_{0}=\left(\epsilon_{s} p_{s} P_{s}\right) \text {. }
$$

The corresponding zeroth order $\nu$-mapping matrix is the $3 \times 3$ real orthogonal matrix

$$
\left(V_{\nu-\text { map }}\right)_{0}=\left(V_{\downarrow}^{\dagger}\right)_{0}\left(V_{\uparrow}\right)_{0} .
$$

By using (1.22)-(1.24), we find

$$
\left(V_{\nu-\text { map }}\right)_{0}=\left(\begin{array}{ccc}
\cos \theta_{\downarrow} \cos \theta_{\uparrow} & -\sin \theta_{\downarrow} \cos \theta_{\uparrow} & \sin \theta_{\uparrow} \sin \phi \\
+\sin \theta_{\downarrow} \sin \theta_{\uparrow} \cos \phi & +\cos \theta_{\downarrow} \sin \theta_{\uparrow} \cos \phi & \\
-\cos \theta_{\downarrow} \sin \theta_{\uparrow} & \sin \theta_{\downarrow} \sin \theta_{\uparrow} & \cos \theta_{\uparrow} \sin \phi \\
+\sin \theta_{\downarrow} \cos \theta_{\uparrow} \cos \phi & +\cos \theta_{\downarrow} \cos \theta_{\uparrow} \cos \phi & \\
-\sin \theta_{\downarrow} \sin \phi & -\cos \theta_{\downarrow} \sin \phi & \cos \phi
\end{array}\right),
$$

in which $\phi_{\downarrow}$ and $\phi_{\uparrow}$ only appear through their difference

$$
\phi=\phi_{\uparrow}-\phi_{\downarrow} .
$$

Eqs. (1.19) and (1.31) account for the three "gauge" degrees of freedom of (1.17).

The above description for the lepton sector corresponds exactly to that for the quark sector in Ref. 1. Their difference lies only in the magnitude of these three angles $\theta_{\downarrow}, \theta_{\uparrow}$ and $\phi$. For quarks, all three angles are small. This is not the case for leptons. Using Eq.(5.14) of Ref. 1, we have

$$
\xi_{\downarrow}=\tan \phi_{\downarrow}=-1
$$

and

$$
\eta_{\uparrow}=\tan \theta_{\uparrow} \cos \phi_{\uparrow}=-\sqrt{\frac{1}{2}}
$$


which are both not small. On the other hand, the parameters

$$
x \equiv \xi_{\uparrow}=\tan \phi_{\uparrow}
$$

and

$$
y \equiv \eta_{\downarrow}=\tan \theta_{\downarrow} \cos \phi_{\downarrow}
$$

are both small. Correspondingly, (1.11) and (1.12) become

$$
\mathcal{M}_{0}\left(l_{\downarrow}\right)=\alpha_{\downarrow}\left|l_{3}(\downarrow)+l_{2}(\downarrow)\right|^{2}+\beta_{\downarrow}\left|l_{2}(\downarrow)-y l_{1}(\downarrow)\right|^{2}+\beta_{\downarrow}\left|l_{3}(\downarrow)+y l_{1}(\downarrow)\right|^{2}
$$

and

$$
\mathcal{M}_{0}\left(l_{\uparrow}\right)=\alpha_{\uparrow}\left|l_{3}(\uparrow)-x l_{2}(\uparrow)\right|^{2}+\beta_{\uparrow}\left|l_{2}(\uparrow)+\sqrt{\frac{1}{2}} l_{1}(\uparrow)\right|^{2}+\beta_{\uparrow}\left|l_{3}(\uparrow)+\sqrt{\frac{1}{2}} x l_{1}(\uparrow)\right|^{2} .
$$

When $x=y=0$, the neutrino mapping matrix $V_{\nu}$ takes on the HarrisonPerkins-Scott form $[5,6]$. In that limit, $x=0$ implies $\phi_{\uparrow}=0$ and therefore

$$
\hat{l}_{3} \| \hat{\nu}_{3} \text {. }
$$

Likewise, when $y=0$, we have $\theta_{\downarrow}=0$ and

$$
\hat{l}_{1} \| \hat{e} \text {. }
$$

In section 2, we discuss the model with $T$ violation and evaluate the masses of $e$ and $\nu_{1}$. The Jarlskog invariant of the neutrino mapping matrix is calculated in section 3 .

\section{2. $T$ violation}

With $T$ violation, we modify (1.34)-(1.35) by writing

$$
\mathcal{M}\left(l_{\downarrow}\right)=\alpha_{\downarrow}\left|l_{3}(\downarrow)+e^{i \chi_{\downarrow}} l_{2}(\downarrow)\right|^{2}+\beta_{\downarrow}\left|l_{2}(\downarrow)-y l_{1}(\downarrow)\right|^{2}+\beta_{\downarrow}\left|l_{3}(\downarrow)+y l_{1}(\downarrow)\right|^{2}
$$

and

$$
\mathcal{M}\left(l_{\uparrow}\right)=\alpha_{\uparrow}\left|l_{3}(\uparrow)-x e^{i \chi_{\uparrow}} l_{2}(\uparrow)\right|^{2}+\beta_{\uparrow}\left|l_{2}(\uparrow)+\sqrt{\frac{1}{2}} l_{1}(\uparrow)\right|^{2}+\beta_{\uparrow}\left|l_{3}(\uparrow)+\sqrt{\frac{1}{2}} x l_{1}(\uparrow)\right|^{2} .
$$


[Note that in Ref. 1, a different choice is made by placing the $T$-violation factor $e^{i \chi \uparrow}$ between $l_{2}(\uparrow)$ and $l_{1}(\uparrow)$.] By using (1.7)-(1.8), the determinants of the corresponding mass matrices are

$$
\left|M\left(l_{\downarrow}\right)\right|=2 \alpha_{\downarrow} \beta_{\downarrow}^{2} y^{2}\left(1-\cos \chi_{\downarrow}\right)
$$

and

$$
\left|M\left(l_{\uparrow}\right)\right|=\alpha_{\uparrow} \beta_{\uparrow}^{2} x^{2}\left(1-\cos \chi_{\uparrow}\right) .
$$

The masses of $e$ and $\nu_{1}$ satisfy

$$
m_{e}\left(m_{e}-\lambda_{\mu}\right)\left(m_{e}-\lambda_{\tau}\right)=\left|M\left(l_{\downarrow}\right)\right|
$$

and

$$
m_{1}\left(m_{1}-\lambda_{2}\right)\left(m_{1}-\lambda_{3}\right)=\left|M\left(l_{\uparrow}\right)\right|
$$

where $\lambda_{\mu}, \lambda_{\tau}, \lambda_{2}$ and $\lambda_{3}$ are the zeroth order masses of $\mu, \tau, \nu_{2}$ and $\nu_{3}$ given by (1.25)-(1.27). Neglecting $m_{e} / m_{\mu}$ and $m_{e} / m_{\tau}$ corrections, (2.5) leads to

$$
m_{e} m_{\mu} m_{\tau} \cong 2 \alpha_{\downarrow} \beta_{\downarrow}^{2} y^{2}\left(1-\cos \chi_{\downarrow}\right) .
$$

Likewise, (1.25)-(1.27) and (1.32)-(1.33) give

$$
m_{\mu} \cong \beta_{\downarrow}\left(1+2 y^{2}\right)
$$

and

$$
m_{\tau} \cong 2 \alpha_{\downarrow}+\beta_{\downarrow} .
$$

Assuming $m_{1} / m_{2}$ and $m_{1} / m_{3}$ are both small (for which there is as yet no experimental evidence), (2.6) gives

$$
m_{1} m_{2} m_{3} \cong \alpha_{\uparrow} \beta_{\uparrow}^{2} x^{2}\left(1-\cos \chi_{\uparrow}\right)
$$

and (1.25)-(1.27), (1.32)-(1.33) give

$$
m_{2} \cong \frac{1}{2} \beta_{\uparrow}\left(3+x^{2}\right)
$$

and

$$
m_{3} \cong \alpha_{\uparrow}\left(1+x^{2}\right)+\beta_{\uparrow} .
$$


It is convenient to introduce in the $\downarrow$ sector a phase transformation of its base-vectors:

$$
\left(\begin{array}{c}
l_{1}(\downarrow) \\
l_{2}(\downarrow) \\
l_{3}(\downarrow)
\end{array}\right)=\Omega_{\downarrow}\left(\begin{array}{c}
e_{1} \\
e_{2} \\
e_{3}
\end{array}\right)
$$

where

$$
\Omega_{\downarrow}=\left(\begin{array}{ccc}
1 & 0 & 0 \\
0 & e^{-i \frac{1}{2} \chi_{\downarrow}} & 0 \\
0 & 0 & e^{i \frac{1}{2} \chi_{\downarrow}}
\end{array}\right)
$$

Hence (2.1) becomes

$$
\begin{aligned}
\mathcal{M}\left(l_{\downarrow}\right)= & \alpha_{\downarrow}\left|e_{3}+e_{2}\right|^{2}+\beta_{\downarrow}\left|e_{2}-y e^{i \chi_{\downarrow} / 2} e_{1}\right|^{2}+\beta_{\downarrow}\left|e_{3}+y e^{-i \chi_{\downarrow} / 2} e_{1}\right|^{2} \\
= & \left(\bar{e}_{1} \bar{e}_{2} \bar{e}_{3}\right)\left[H_{0}(\downarrow)+H_{1}(\downarrow)+O\left(y^{2}\right)\right]\left(\begin{array}{c}
e_{1} \\
e_{2} \\
e_{3}
\end{array}\right)
\end{aligned}
$$

with

$$
\begin{gathered}
H_{0}(\downarrow)=\left(\begin{array}{ccc}
0 & 0 & 0 \\
0 & \alpha_{\downarrow}+\beta_{\downarrow} & \alpha_{\downarrow} \\
0 & \alpha_{\downarrow} & \alpha_{\downarrow}+\beta_{\downarrow}
\end{array}\right) \\
H_{1}(\downarrow)=\beta_{\downarrow} y\left(\begin{array}{ccc}
0 & -e^{-i \frac{1}{2} \chi_{\downarrow}} & e^{i \frac{1}{2} \chi_{\downarrow}} \\
-e^{i \frac{1}{2} \chi_{\downarrow}} & 0 & 0 \\
e^{-i \frac{1}{2} \chi_{\downarrow}} & 0 & 0
\end{array}\right) .
\end{gathered}
$$

Likewise, we write (2.2) as

$$
\mathcal{M}\left(l_{\uparrow}\right)=\left(\bar{l}_{1}(\uparrow) \bar{l}_{2}(\uparrow) \bar{l}_{3}(\uparrow)\right)\left[H_{0}(\uparrow)+H_{1}(\uparrow)+O\left(x^{2}\right)\right]\left(\begin{array}{c}
l_{1}(\uparrow) \\
l_{2}(\uparrow) \\
l_{3}(\uparrow)
\end{array}\right)
$$

where

$$
H_{0}(\uparrow)=\left(\begin{array}{ccc}
\frac{1}{2} \beta_{\uparrow} & \sqrt{\frac{1}{2}} \beta_{\uparrow} & 0 \\
\sqrt{\frac{1}{2}} \beta_{\uparrow} & \beta_{\uparrow} & 0 \\
0 & 0 & \alpha_{\uparrow}+\beta_{\uparrow}
\end{array}\right)
$$


and

$$
H_{1}(\uparrow)=x\left(\begin{array}{ccc}
0 & 0 & \sqrt{\frac{1}{2}} \beta_{\uparrow} \\
0 & 0 & -\alpha_{\uparrow} e^{-i \chi_{\uparrow}} \\
\sqrt{\frac{1}{2}} \beta_{\uparrow} & -\alpha_{\uparrow} e^{i \chi_{\uparrow}} & 0
\end{array}\right) .
$$

The matrices $H_{0}(\downarrow)$ and $H_{0}(\uparrow)$ can be readily diagonalized:

$$
\begin{aligned}
& \mathcal{V}_{\downarrow}^{\dagger} H_{0}(\downarrow) \mathcal{V}_{\downarrow}=\left(\begin{array}{ccc}
0 & 0 & 0 \\
0 & m_{0}(\mu) & 0 \\
0 & 0 & m_{0}(\tau)
\end{array}\right) \\
& \mathcal{V}_{\uparrow}^{\dagger} H_{0}(\uparrow) \mathcal{V}_{\uparrow}=\left(\begin{array}{ccc}
0 & 0 & 0 \\
0 & m_{0}(2) & 0 \\
0 & 0 & m_{0}(3)
\end{array}\right)
\end{aligned}
$$

where

$$
\begin{aligned}
& m_{0}(\mu)=\beta_{\downarrow}, \quad m_{0}(\tau)=2 \alpha_{\downarrow}+\beta_{\downarrow}, \\
& m_{0}(2)=\frac{3}{2} \beta_{\uparrow}, \quad m_{0}(3)=\alpha_{\uparrow}+\beta_{\uparrow}
\end{aligned}
$$

and

$$
\mathcal{V}_{\downarrow}=(\hat{\epsilon} \hat{m} \hat{t}), \quad \mathcal{V}_{\uparrow}=\left(\hat{n}_{1} \hat{n}_{2} \hat{n}_{3}\right)
$$

with

$$
\hat{\epsilon}=\left(\begin{array}{l}
1 \\
0 \\
0
\end{array}\right), \quad \hat{m}=\sqrt{\frac{1}{2}}\left(\begin{array}{r}
0 \\
1 \\
-1
\end{array}\right), \quad \hat{t}=\sqrt{\frac{1}{2}}\left(\begin{array}{l}
0 \\
1 \\
1
\end{array}\right)
$$

and

$$
\hat{n}_{1}=\left(\begin{array}{r}
\sqrt{\frac{2}{3}} \\
-\sqrt{\frac{1}{3}} \\
0
\end{array}\right), \quad \hat{n}_{2}=\left(\begin{array}{c}
\sqrt{\frac{1}{3}} \\
\sqrt{\frac{2}{3}} \\
0
\end{array}\right), \quad \hat{n}_{3}=\left(\begin{array}{l}
0 \\
0 \\
1
\end{array}\right) .
$$

Correspondingly, define

$$
h_{\downarrow} \equiv \mathcal{V}_{\downarrow}^{\dagger} H_{1}(\downarrow) \mathcal{V}_{\downarrow} \text { and } h_{\uparrow} \equiv \mathcal{V}_{\uparrow}^{\dagger} H_{1}(\uparrow) \mathcal{V}_{\uparrow} .
$$

We find

$$
h_{\downarrow}=\sqrt{2} \beta_{\downarrow} y\left(\begin{array}{ccc}
0 & -\cos \frac{1}{2} \chi_{\downarrow} & i \sin \frac{1}{2} \chi_{\downarrow} \\
-\cos \frac{1}{2} \chi_{\downarrow} & 0 & 0 \\
-i \sin \frac{1}{2} \chi_{\downarrow} & 0 & 0
\end{array}\right)
$$


and

$$
h_{\uparrow}=x\left(\begin{array}{ccc}
0 & 0 & A^{*} \\
0 & 0 & B^{*} \\
A & B & 0
\end{array}\right)
$$

where

$$
A=\sqrt{\frac{1}{3}}\left(\beta_{\uparrow}+\alpha_{\uparrow} e^{i \chi_{\uparrow}}\right)
$$

and

$$
B=\sqrt{\frac{1}{6}}\left(\beta_{\uparrow}-2 \alpha_{\uparrow} e^{i \chi_{\uparrow}}\right) .
$$

Represent $\hat{\epsilon}, \hat{m}, \hat{t}$ and $\hat{n}_{1}, \hat{n}_{2}, \hat{n}_{3}$ of (2.26)-(2.27) by their ket-vectors (in Dirac's notation)

$$
\mid \epsilon), \mid m), \mid t)
$$

and

$$
\left.\left.\left.\mid n_{1}\right), \mid n_{2}\right), \mid n_{3}\right)
$$

Correspondingly, we designate

$$
\mid e), \mid \mu), \mid \tau)
$$

and

$$
\left.\left.\left.\mid \nu_{1}\right), \mid \nu_{2}\right), \mid \nu_{3}\right)
$$

to be the physical lepton states. Introduce the transformation matrices

$$
\mathcal{W}_{\downarrow}=\equiv\left(\begin{array}{ccc}
(\epsilon \mid e) & (\epsilon \mid \mu) & (\epsilon \mid \tau) \\
(m \mid e) & (m \mid \mu) & (m \mid \tau) \\
(t \mid e) & (t \mid \mu) & (t \mid \tau)
\end{array}\right)
$$

and

$$
\mathcal{W}_{\uparrow}=\equiv\left(\begin{array}{lll}
\left(n_{1} \mid \nu_{1}\right) & \left(n_{1} \mid \nu_{2}\right) & \left(n_{1} \mid \nu_{3}\right) \\
\left(n_{2} \mid \nu_{1}\right) & \left(n_{2} \mid \nu_{2}\right) & \left(n_{2} \mid \nu_{3}\right) \\
\left(n_{3} \mid \nu_{1}\right) & \left(n_{3} \mid \nu_{2}\right) & \left(n_{3} \mid \nu_{3}\right)
\end{array}\right) .
$$

To first order perturbation in $x$ and $y$, we find

$$
\mathcal{W}_{\downarrow}=1+\sqrt{2} \beta_{\downarrow} y\left(\begin{array}{ccc}
0 & -\frac{1}{m_{0}(\mu)} \cos \frac{1}{2} \chi_{\downarrow} & \frac{i}{m_{0}(\tau)} \sin \frac{1}{2} \chi_{\downarrow} \\
\frac{1}{m_{0}(\mu)} \cos \frac{1}{2} \chi_{\downarrow} & 0 & 0 \\
\frac{i}{m_{0}(\tau)} \sin \frac{1}{2} \chi_{\downarrow} & 0 & 0
\end{array}\right)
$$


and

$$
\mathcal{W}_{\uparrow}=1+x\left(\begin{array}{ccc}
0 & 0 & \frac{A^{*}}{\alpha_{\uparrow}+\beta_{\uparrow}} \\
0 & 0 & \frac{2 B^{*}}{2 \alpha_{\uparrow}-\beta_{\uparrow}} \\
-\frac{A}{\alpha_{\uparrow}+\beta_{\uparrow}} & -\frac{2 B}{2 \alpha_{\uparrow}-\beta_{\uparrow}} & 0
\end{array}\right) .
$$

Denote $U_{\downarrow}$ and $U_{\uparrow}$ to be the unitary matrices that diagonalize the mass matrices $M\left(l_{\downarrow}\right)$ and $M\left(l_{\uparrow}\right)$ defined by (1.7)-(1.8) and (2.1)-(2.2). To first order in $x$ and $y$, we have

$$
U_{\downarrow}=\Omega_{\downarrow} \mathcal{V}_{\downarrow} \mathcal{W}_{\downarrow}
$$

and

$$
U_{\uparrow}=\mathcal{V}_{\uparrow} \mathcal{W}_{\uparrow} .
$$

Combining with $\Omega_{\downarrow}$ given by (2.14), $\mathcal{V}_{\downarrow}, \mathcal{V}_{\uparrow}$ by $(2.25)$ and $\mathcal{W}_{\downarrow}, \mathcal{W}_{\uparrow}$ by $(2.37)-$ (2.38), we derive

$$
U_{\downarrow}=\left(\begin{array}{ccc}
1 & -y X & i y Y \\
\frac{y Z}{\sqrt{2}} e^{-i \frac{1}{2} \chi_{\downarrow}} & \frac{1}{\sqrt{2}} e^{-i \frac{1}{2} \chi_{\downarrow}} & \frac{1}{\sqrt{2}} e^{-i \frac{1}{2} \chi_{\downarrow}} \\
-\frac{y Z^{*}}{\sqrt{2}} e^{i \frac{1}{2} \chi_{\downarrow}} & -\frac{1}{\sqrt{2}} e^{i \frac{1}{2} \chi_{\downarrow}} & \frac{1}{\sqrt{2}} e^{i \frac{1}{2} \chi_{\downarrow}}
\end{array}\right)
$$

with

$$
X=\sqrt{2} \cos \frac{1}{2} \chi_{\downarrow}, \quad Y=\frac{\sqrt{2} \beta_{\downarrow}}{2 \alpha_{\downarrow}+\beta_{\downarrow}} \sin \frac{1}{2} \chi_{\downarrow}
$$

and

$$
Z=X+i Y
$$

Likewise,

$$
U_{\uparrow}=\left(\begin{array}{ccc}
\sqrt{\frac{2}{3}} & \sqrt{\frac{1}{3}} & x \sqrt{\frac{1}{3}}\left(\frac{\sqrt{2} A^{*}}{\alpha_{\uparrow}+\beta_{\uparrow}}+\frac{2 B^{*}}{2 \alpha_{\uparrow}-\beta_{\uparrow}}\right) \\
-\sqrt{\frac{1}{3}} & \sqrt{\frac{2}{3}} & x \sqrt{\frac{1}{3}}\left(\frac{-A^{*}}{\alpha_{\uparrow}+\beta_{\uparrow}}+\frac{2 \sqrt{2} B^{*}}{2 \alpha_{\uparrow}-\beta_{\uparrow}}\right) \\
-\frac{x A}{\alpha_{\uparrow}+\beta_{\uparrow}} & -\frac{2 x B}{2 \alpha_{\uparrow}-\beta_{\uparrow}} & 1
\end{array}\right)
$$

with $A$ and $B$ given by (2.31) and (2.32). The neutrino mapping matrix $U_{\nu-\text { map }}$ is then related to $(2.41)$ and $(2.44)$ by

$$
U_{\nu-\text { map }}=U_{\downarrow}^{\dagger} U_{\uparrow} .
$$

Note that in accordance with our definitions (2.39)-(2.40) $U_{\uparrow}$ and $U_{\downarrow}$ refer to the transformation matrices relating the "bare" annihilation operators 
$l_{i}(\downarrow), l_{i}(\uparrow)$ defined by the mass operators (1.7)-(1.8) to the corresponding "physical" annihilation operators of leptons (not their state vectors). Hence, the $\nu$-mapping matrix in the particle data group literature is $U_{\nu-\text { map }}^{*}$, the complex conjugate of (2.45).

\section{Jarlskog Invariant}

The matrix $U_{\nu-m a p}$ can be written as

$$
U_{\nu-m a p}=\left(\begin{array}{ccc}
U_{e 1} & U_{e 2} & U_{e 3} \\
U_{\mu 1} & U_{\mu 2} & U_{\mu 3} \\
U_{\tau 1} & U_{\tau 2} & U_{\tau 3}
\end{array}\right) .
$$

From (2.41), (2.44)-(2.45) and to first order in $x$ and $y$, we find

$$
\begin{gathered}
U_{e 1}=\sqrt{\frac{2}{3}}-y \sqrt{\frac{1}{6}} Z^{*} e^{i \frac{1}{2} \chi_{\downarrow}} \\
U_{e 2}=\sqrt{\frac{1}{3}}+y \sqrt{\frac{1}{3}} Z^{*} e^{i \frac{1}{2} \chi_{\downarrow}} \\
U_{e 3}=x \sqrt{\frac{1}{3}}\left(\frac{\sqrt{2} A^{*}}{\alpha_{\uparrow}+\beta_{\uparrow}}+\frac{2 B^{*}}{2 \alpha_{\uparrow}-\beta_{\uparrow}}\right)-y \sqrt{\frac{1}{2}} Z e^{-i \frac{1}{2} \chi_{\downarrow}} \\
U_{\mu 1}=-y \sqrt{\frac{2}{3}} X-\sqrt{\frac{1}{6}} e^{i \frac{1}{2} \chi_{\downarrow}}+x \sqrt{\frac{1}{2}} \frac{A}{\alpha_{\uparrow}+\beta_{\uparrow}} e^{-i \frac{1}{2} \chi_{\downarrow}} \\
U_{\mu 2}=-y \sqrt{\frac{1}{3}} X+\sqrt{\frac{1}{3}} e^{i \frac{1}{2} \chi_{\downarrow}}+x \frac{\sqrt{2} B}{2 \alpha_{\uparrow}-\beta_{\uparrow}} e^{-i \frac{1}{2} \chi_{\downarrow}} \\
U_{\mu 3}=x \sqrt{\frac{1}{6}}\left(-\frac{A A^{*}}{\alpha_{\uparrow}+\beta_{\uparrow}}+\frac{2 \sqrt{2} B^{*}}{2 \alpha_{\uparrow}-\beta_{\uparrow}}\right) e^{i \frac{1}{2} \chi_{\downarrow}}-\sqrt{\frac{1}{2}} e^{-i \frac{1}{2} \chi_{\downarrow}} \\
U_{\tau 1}=-i y \sqrt{\frac{2}{3}} Y-\sqrt{\frac{1}{6}} e^{i \frac{1}{2} \chi_{\downarrow}}-x \sqrt{\frac{1}{2}} \frac{A}{\alpha_{\uparrow}+\beta_{\uparrow}} e^{-i \frac{1}{2} \chi_{\downarrow}} \\
U_{\tau 2}=-i y \sqrt{\frac{1}{3}} Y+\sqrt{\frac{1}{3}} e^{i \frac{1}{2} \chi_{\downarrow}}-x \frac{\sqrt{2} B}{2 \alpha_{\uparrow}-\beta_{\uparrow}} e^{-i \frac{1}{2} \chi_{\downarrow}}
\end{gathered}
$$


and

$$
U_{\tau 3}=x \sqrt{\frac{1}{6}}\left(-\frac{A^{*}}{\alpha_{\uparrow}+\beta_{\uparrow}}+\frac{2 \sqrt{2} B^{*}}{2 \alpha_{\uparrow}-\beta_{\uparrow}}\right) e^{i \frac{1}{2} \chi_{\downarrow}}+\sqrt{\frac{1}{2}} e^{-i \frac{1}{2} \chi_{\downarrow}}
$$

where $A, B, X, Y$ and $Z$ are given by (2.31)-(2.32) and (2.42)-(2.43).

Define

$$
T_{1}=U_{e 1}^{*} U_{\mu 1}, \quad T_{2}=U_{e 2}^{*} U_{\mu 2} \text { and } T_{3}=U_{e 3}^{*} U_{\mu 3} .
$$

By using (3.2), we have

$$
\begin{aligned}
& T_{1}=-\frac{1}{3} e^{i \frac{1}{2} \chi_{\downarrow}}-y \frac{2}{3} X+x \sqrt{\frac{1}{3}} \frac{A}{\alpha_{\uparrow}+\beta_{\uparrow}} e^{-i \frac{1}{2} \chi_{\downarrow}}+y \frac{1}{6} Z, \\
& T_{2}=\frac{1}{3} e^{i \frac{1}{2} \chi_{\downarrow}}-y \frac{1}{3} X+x \sqrt{\frac{2}{3}} \frac{B}{2 \alpha_{\uparrow}-\beta_{\uparrow}} e^{-i \frac{1}{2} \chi_{\downarrow}}+y \frac{1}{3} Z
\end{aligned}
$$

and

$$
T_{3}=-x \sqrt{\frac{1}{3}}\left(\frac{A}{\alpha_{\uparrow}+\beta_{\uparrow}}+\frac{\sqrt{2} B}{2 \alpha_{\uparrow}-\beta_{\uparrow}}\right) e^{-i \frac{1}{2} \chi_{\downarrow}}+y \frac{1}{2} Z^{*} .
$$

Thus,

$$
T_{1}+T_{2}+T_{3}=0
$$

The Jarlskog invariant $J_{\nu-\text { map }}$ for the neutrino mapping matrix is given by $\operatorname{Im} T_{1}^{*} T_{2}$. We find

$J_{\nu-\text { map }}=-\frac{y}{6 \sqrt{2}}\left(1+\frac{\beta_{\downarrow}}{2 \alpha_{\downarrow}+\beta_{\downarrow}}\right) \sin \chi_{\downarrow}+\frac{\alpha_{\uparrow} \beta_{\uparrow} x}{3\left(\alpha_{\uparrow}+\beta_{\uparrow}\right)\left(2 \alpha_{\uparrow}-\beta_{\uparrow}\right)}\left[\sin \chi_{\downarrow}+\sin \left(\chi_{\uparrow}-\chi_{\downarrow}\right)\right]$

which is valid for small $x$ and $y$. If in addition $\chi_{\downarrow}$ and $\chi_{\uparrow}$ are also small, then

$$
J_{\nu-\text { map }} \cong-\frac{y \chi_{\downarrow}}{6 \sqrt{2}}\left(1+\frac{\beta_{\downarrow}}{2 \alpha_{\downarrow}+\beta_{\downarrow}}\right)+\frac{x \chi_{\uparrow} \alpha_{\uparrow} \beta_{\uparrow}}{3\left(\alpha_{\uparrow}+\beta_{\uparrow}\right)\left(2 \alpha_{\uparrow}-\beta_{\uparrow}\right)} .
$$

From (2.7) and (2.23) we find

$$
m_{\mu} \cong \beta_{\downarrow}, \quad m_{\tau} \cong 2 \alpha_{\downarrow}+\beta_{\downarrow}
$$

and

$$
m_{e} m_{\mu} m_{\tau} \cong \alpha_{\downarrow} \beta_{\downarrow}^{2} y^{2} \chi_{\downarrow}^{2}
$$


Thus,

$$
y \chi_{\downarrow} \cong \pm \sqrt{\frac{2 m_{e} m_{\tau}}{m_{\mu}\left(m_{\tau}-m_{\mu}\right)}} .
$$

Likewise, from (2.10) and (2.24)

$$
m_{2} \cong \frac{3}{2} \beta_{\uparrow}, \quad m_{3} \cong \alpha_{\uparrow}+\beta_{\uparrow}
$$

and

$$
m_{1} m_{2} m_{3} \cong \frac{1}{2} \alpha_{\uparrow} \beta_{\uparrow}^{2} x^{2} \chi_{\uparrow}^{2},
$$

which lead to

$$
x \chi_{\uparrow} \cong \pm 3 \sqrt{\frac{3 m_{1} m_{3}}{2 m_{2}\left(3 m_{3}-2 m_{2}\right)}} .
$$

Write (3.7) as

$$
J_{\nu-m a p}=J_{e}+J_{\nu}
$$

in which

$$
\begin{aligned}
J_{e} & =\frac{1}{6} \sqrt{\frac{m_{e}}{m_{\mu}}}\left[\frac{m_{\tau}+m_{\mu}}{\sqrt{m_{\tau}\left(m_{\tau}-m_{\mu}\right)}}\right] \\
& =\frac{1}{6}\left[\sqrt{\frac{m_{e}}{m_{\mu}}}+O\left(\sqrt{\frac{m_{e} m_{\mu}}{m_{\tau}^{2}}}\right)\right]
\end{aligned}
$$

and if $m_{1} / m_{2}$ and $m_{2} / m_{3}$ are both $<<1$ then

$$
J_{\nu} \cong \pm \frac{1}{3 \sqrt{2}} \sqrt{\frac{m_{1} m_{2}}{m_{3}^{2}}} .
$$

For convenience, we set the sign of $J_{e}$ to be positive, thus,

$$
J_{\nu-\text { map }} \cong \frac{1}{6} \sqrt{\frac{m_{e}}{m_{\mu}}}\left[\frac{m_{\tau}+m_{\mu}}{\sqrt{m_{\tau}\left(m_{\tau}-m_{\mu}\right)}}\right] \pm \frac{1}{3 \sqrt{2}} \sqrt{\frac{m_{1} m_{2}}{m_{3}^{2}}}
$$

which leads to (1.2). 


\section{Appendix}

Consider the eigenstate equation

$$
\left.H \mid i)=m_{i} \mid i\right)
$$

of a $3 \times 3$ hermitian Hamiltonian

$$
H=H_{0}+h
$$

with $i=1,2,3$ and $H_{0}$ diagonal. In the case that the eigenvalues $m_{i}$ are known (or can be derived by a simple series expansion as in the case of the masses of $e, \mu, \tau$ and $\nu_{1}, \nu_{2}, \nu_{3}$ through (2.5)-(2.6)), the explicit form of the eigenvectors $\mid i$ ) can be obtained by solving a simple linear equation of two variables, as we shall see. Write

$$
H_{0}=\left(\begin{array}{ccc}
n_{\xi} & 0 & 0 \\
0 & n_{\eta} & 0 \\
0 & 0 & n_{\zeta}
\end{array}\right)
$$

and

$$
h=\left(\begin{array}{ccc}
h_{\xi \xi} & h_{\xi \eta} & h_{\xi \zeta} \\
h_{\eta \xi} & h_{\eta \eta} & h_{\eta \zeta} \\
h_{\zeta \xi} & h_{\zeta \eta} & h_{\zeta \zeta}
\end{array}\right) .
$$

Denote the normalized eigenstates of $H_{0}$ by $\mid s$ ), so that

$$
\left.\left.H_{0} \mid s\right)=n_{s} \mid s\right) \text {. }
$$

with $s=\xi, \eta, \zeta$ and the normalization

$$
(\xi \mid \xi)=(\eta \mid \eta)=(\zeta \mid \zeta)=1 .
$$

However, for the eigenstates of $H$ we choose the Brillouin-Wigner normalization condition with

$$
(\xi \mid 1)=(\eta \mid 2)=(\zeta \mid 3)=1 .
$$

The three eigenstates $\mid i)$ of (A.1) can then be written as

$$
\mid 1)=\mid \xi)+(\eta \mid 1) \mid \eta)+(\zeta \mid 1) \mid \zeta)
$$




$$
\begin{aligned}
& \mid 2)=(\xi \mid 2) \mid \xi)+\mid \eta)+(\zeta \mid 2) \mid \zeta) \\
& \mid 3)=(\xi \mid 3) \mid \xi)+(\eta \mid 3) \mid \eta)+\mid \zeta)
\end{aligned}
$$

Since

$$
\left.H \mid 1)=m_{1} \mid 1\right)
$$

we have

$$
(\xi|H| 1)=m_{1}(\xi \mid 1)=m_{1} .
$$

On the other hand,

$$
\left.\left.H \mid 1)=H_{0} \mid 1\right)+h \mid 1\right)
$$

which gives

$$
(\xi|H| 1)=n_{\xi}(\xi \mid 1)+(\xi|h| 1)=n_{\xi}+(\xi|h| 1) .
$$

Thus, (A.10) and (A.12) yield

$$
m_{1}=n_{\xi}+(\xi|h| 1) .
$$

Likewise, from (A.9) we find

$$
(\eta|H| 1)=m_{\eta}(\eta \mid 1)
$$

and by using (A.11)

$$
(\eta|H| 1)=n_{\eta}(\eta \mid 1)+(\eta|h| 1) .
$$

Combining (A.14) and (A.15), we derive

$$
(\eta \mid 1)=\frac{1}{m_{1}-m_{\eta}}(\eta|h| 1)
$$

and, in identical way,

$$
(\zeta \mid 1)=\frac{1}{m_{1}-m_{\zeta}}(\zeta|h| 1) .
$$

Next, introduce

$$
x=(\eta \mid 1), \quad y=(\zeta \mid 1)
$$

and write the first equation in (A.8) as

$$
\mid 1)=\mid \xi)+x \mid \eta)+y \mid \zeta) .
$$


This leads to

$$
h \mid 1)=h \mid \xi)+x h \mid \eta)+y h \mid \zeta)
$$

therefore

$$
(\eta|h| 1)=h_{\eta \xi}+x h_{\eta \eta}+y h_{\eta \zeta}
$$

and

$$
(\zeta|h| 1)=h_{\zeta \xi}+x h_{\zeta \eta}+y h_{\zeta \zeta}
$$

From (A.16), (A.18) and (A.21) it follows

$$
x=\frac{1}{m_{1}-m_{\eta}}\left[h_{\eta \xi}+x h_{\eta \eta}+y h_{\eta \zeta}\right] .
$$

Likewise, from (A.17) and (A.22),

$$
y=\frac{1}{m_{1}-m_{\zeta}}\left[h_{\zeta \xi}+x h_{\zeta \eta}+y h_{\zeta \zeta}\right] .
$$

Define

$$
\Delta(1)=\left(m_{1}-n_{\eta}-h_{\eta \eta}\right)\left(m_{1}-n_{\zeta}-h_{\zeta \zeta}\right)-h_{\eta \zeta} h_{\zeta \eta} .
$$

By using (A.18) and (A.23)-(A.24), we find

$$
(\eta \mid 1)=\frac{1}{\Delta(1)}\left[\left(m_{1}-n_{\zeta}-h_{\zeta \zeta}\right) h_{\eta \xi}+h_{\eta \zeta} h_{\zeta \xi}\right]
$$

and

$$
(\zeta \mid 1)=\frac{1}{\Delta(1)}\left[\left(m_{1}-n_{\eta}-h_{\eta \eta}\right) h_{\zeta \xi}+h_{\zeta \eta} h_{\eta \xi}\right] .
$$

Thus we have the explicit solution of $\mid 1$, with the Brillouin-Wigner normalization introduced in (A.8). Likewise, the other two eigenstates |2) and |3) can be similarly derived. We find

$$
\begin{aligned}
(\zeta \mid 2) & =\frac{1}{\Delta(2)}\left[\left(m_{2}-n_{\xi}-h_{\xi \xi}\right) h_{\zeta \eta}+h_{\zeta \xi} h_{\xi \eta}\right], \\
(\xi \mid 2) & =\frac{1}{\Delta(2)}\left[\left(m_{2}-n_{\zeta}-h_{\zeta \zeta}\right) h_{\xi \eta}+h_{\xi \zeta} h_{\zeta \eta}\right], \\
(\xi \mid 3) & =\frac{1}{\Delta(3)}\left[\left(m_{3}-n_{\eta}-h_{\eta \eta}\right) h_{\xi \zeta}+h_{\xi \eta} h_{\eta \zeta}\right]
\end{aligned}
$$


and

$$
(\eta \mid 3)=\frac{1}{\Delta(3)}\left[\left(m_{3}-n_{\xi}-h_{\xi \xi}\right) h_{\eta \zeta}+h_{\eta \xi} h_{\xi \zeta}\right]
$$

with

$$
\Delta(2)=\left(m_{2}-n_{\zeta}-h_{\zeta \zeta}\right)\left(m_{2}-n_{\xi}-h_{\xi \xi}\right)-h_{\zeta \xi} h_{\xi \zeta}
$$

and

$$
\Delta(3)=\left(m_{3}-n_{\xi}-h_{\xi \xi}\right)\left(m_{3}-n_{\eta}-h_{\eta \eta}\right)-h_{\xi \eta} h_{\eta \xi} .
$$

These formulas would be useful for higher order corrections to $U_{\nu-\text { map }}$ and $J_{\nu-\text { map }}$.

References

[1] R. Friedberg and T. D. Lee, Ann. Phys. in press

[2] S. Eidelman et al. (Particle Data Group), Phys. Lett. B592, 1(2004).

[3] T. D. Lee, Phys. Rev. D8, 1226(1973)

[4] T. D. Lee, Physics Reports 9c, 2(1974)

[5] P. F. Harrison, D. H. Perkins and W. G. Scott, Phys. Lett. B530, 167(2002).

[6] L. Wolfenstein, Phys. Rev. D18, 958(1978);

P. F. Harrison and W. G. Scott, Phys. Lett. B535, 163(2002);

Z. Z. Xing, Phys. Lett. B533, 85(2002);

X. G. He and A. Zee, Phys. Lett. B560, 87(2003). 